\title{
Curva ambiental de Kuznets e desenvolvimento econômico sustentável
}

\author{
Ronaldo A. Arraes* \\ Marcelo B. Diniz** \\ Márcia J. T. Diniz***
}

Resumo: A motivação deste artigo se baseia na controvérsia existente na literatura recente sobre crescimento econômico, desenvolvimento sustentável e proteção ambiental, gerada a partir da evidência empírica mostrada inicialmente por Grossman e Krueger $(1995,1996)$, onde a relação entre PIB per capita e emissão de poluentes toma a forma de um U-invertido, denominada na literatura como Curva Ambiental de Kuznets (CAK). Este artigo se distingue por contribuir com a explicação de fatores ligados ao desenvolvimento econômico. Como essa relação tem sido recentemente contestada, muitas interpretações estruturais da CAK têm permanecido fortemente sob o amparo ad hoc. A questão sobre tal fato estilizado é se o crescimento econômico gera por si só uma proteção automática ao meio ambiente, vis a vis ao desenvolvimento sustentável. A partir de dados em painel para países, verifica-se que as variáveis que denotam desenvolvimento sustentável apresentam uma relação fraca com o PIB per capita, quanto a sua representação para uma CAK. Há evidências para curvas ambientais com formato cúbico, indicando que o fenômeno pode ser cíclico, rejeitando-se assim as CAK originais, além de todos os indicadores de desenvolvimento selecionados apontarem para divergências entre países, exceção apenas para o indicador relacionado à educação.

* CAEN / UFC, CE. ronal@ufc.br

** UFPA, PA

*** NAEA / UFPA, PA 
Palavras-chave: desenvolvimento sustentável, curva ambiental de Kuznets, países.

Classificação JEL: Q01, O15, O57

Abstract: The motivation of this article bases on the controversy in the recent literature about economic growth, sustainable development and environmental protection, started with the empirical evidences presented by Grossman and Krueger (1995, 1996), where the relationship between per capita GDP and emission of pollutants takes the shape of an inverted-U, denominated in the literature as Environmental Kuznets Curves (EKC). This article differs from others by contributing with further explanation stemming from economic development indicators. Despite being contested by many authors, several structural interpretations of EKC have been strongly sustained under ad hoc shield. The concern about such stylized fact is whether or not the economic growth itself generates an automatic protection to the environment, consequently to the maintainable development. Based upon panel data for countries, it is verified that the variables that denote sustainable economic development present a weak relationship with per capita GDP to support an EKC representation. There are also evidences for environmental curves in cubic format, which means rejection of EKC, besides most of the development indicators pointing out for divergences among countries.

Key words: sustainable economic development, environmental Kuznets curve, countries.

JEL: Q01, O15, O57

\section{Introdução}

A relação entre crescimento econômico e distribuição da renda pessoal em formato de "U-invertido", observada inicialmente por Kuznets (1955) para os EUA, Grã-Bretanha e Alemanha, tem sua explicação fundamentada essencialmente no diferencial de rendimentos na transição de uma economia agrícola para uma economia industrial, como reflexo 
da diferença dos produtos marginais do trabalho entre os dois setores. A idéia básica era que a distribuição de renda tem que ser pensada como uma combinação da distribuição de renda da população rural e urbana, onde esta última, apesar de deter uma renda média mais alta, apresenta maior concentração do que a primeira. Além do mais, quando a renda cresce o diferencial de renda per capita entre as áreas rural e urbana persistiria, ou mesmo alargaria, uma vez que o crescimento da produtividade na área urbana seria maior que na área rural.

Entretanto, Kuznets acreditava que a desigualdade eventualmente seria levada para baixo, por três motivos (Grossman, 2000). Primeiro, porque os indivíduos com altas habilidades seriam também aqueles com altos níveis de renda, de modo que não haveria razão para que seus descendentes tivessem talento o bastante para auferirem altos ganhos. Segundo, porque a população urbana nova, imigrante procedente da zona rural ou do exterior teria condições de tirar menos vantagem das possibilidades da vida na cidade e, portanto, de se apropriar de uma maior parcela da renda, em relação ao que foi possível para a população que imigrou inicialmente. Terceiro, acreditava Kuznets que em sociedades democráticas haveria uma demanda crescente para redistribuição da renda quando a economia crescesse, especialmente porque essas sociedades experimentariam a ampliação do poder político dos grupos urbanos de baixa renda.

A despeito da explicação dada por Kuznets ser bem intuitiva, e mesmo recorrente para explicação do percurso histórico de alguns países em desenvolvimento, na década de 1990 surgiram várias evidências empíricas em contrário ensejando, por sua vez, explicações teóricas que sustentavam formatos da relação entre desigualdade e crescimento diferentes daquela prevista por Kuznets. Apesar dessa controvérsia, também na década de 1990, a curva de Kuznets passou a ser referência para explicar a relação de como a poluição ambiental em suas várias formas evolui em razão do crescimento econômico. O formato da curva é explicado através de dois argumentos. A porção ascendente reflete o progresso natural do desenvolvimento econômico, passando-se de uma economia agrária "limpa" para uma economia industrial "poluída” e para uma economia de serviços "limpos" (Arrow et al, 1995). Enquanto a porção descendente seria o mecanismo das economias desenvolvidas 
exportarem processos de produção intensivos em poluição para economias menos desenvolvidas (Suri; Chapman, 1998). Daí, alguns autores, como Beckerman (1992), defenderem que, ao longo do processo de crescimento, a melhor, senão a única, maneira para se atingir qualidade ambiental na maioria dos países é tornando-se rico.

Não obstante, da constatação inicial de Grossman e Krueger (1993) para os EUA, recentemente tem-se buscado evidências empíricas sobre se a interação entre crescimento econômico e problemas ambientais guarda uma certa regularidade e exibe, também, uma relação de "U-invertido" ou Curva Ambiental de Kuznets (CAK), como assim passou a ser denominada. Algumas evidências têm dado suporte à existência da CAK; por exemplo, Grossman; Krueger (1995), Hilton; Levinson (1998), Khan (1998) sustentam a comprovação do tradicional formato em U-invertido. Por outro lado, Jones; Manuelli (1995) se baseiam em modelos de geração superpostas que, dependendo de decisões institucionais o formato da curva poderia ser monotonicamente crescente ou até senoidal, enquanto Brock; Taylor (2004), adaptando o modelo de crescimento de Solow admite que, para os Estados Unidos, a curva poderia ser monotonicamente decrescente. Já Stokey (1998) defende a hipótese da existência, ao longo do crescimento econômico, de um ponto limite a partir do qual somente tecnologias "limpas" são usadas, demonstrando o formato da CAK como V-invertido, sendo o ápice tal ponto limite.

Destarte que o interesse despertado pela curva ambiental de Kuznet em estudos cross country ou em dados em painel, está no fato de seu formato revelar uma tendência a convergência, especialmente dos países em desenvolvimento, a gerar indicadores ambientais similares aos dos países desenvolvidos, cujas leis e regulamentações ambientais, bem como a consciência ambiental dos seus cidadãos, estariam em um patamar muito mais adiantado do que aqueles primeiros. Afinal, se existe um processo de "convergência ambiental” em curso, então, os esforços multilaterais empreendidos, especialmente pela ONU e Banco Mundial, estariam no caminho certo de resolver a "crise ambiental" vislumbrada desde a década de 1980. No entanto, assim como a relação que lhe deu origem não é plenamente aceita, as explicações teóricas e evidências empíricas entre crescimento e poluição ambiental também não é consensual. A nível teórico, as explicações que sustentam a cur- 
va de Kuznets não poderiam aqui ser aplicadas empiricamente, sendo especialmente difícil de se explicar sua porção descendente, sem antes testar sua possível trajetória cíclica.

Assim, oriundo da discussão dos fatores causais que levam a existência de um relacionamento entre problemas ambientais e crescimento econômico é que este trabalho volta-se para dois objetivos, os quais somam contribuições ao assunto. O primeiro é apresentar para uma análise cross-country em painel, novas evidências empíricas que confirmam a existência de movimento cíclico na curva ambiental de Kuznet, e o segundo é verificar se essa evidência se replica para outras "variáveis ambientais", pelo menos do ponto de vista das proposições do desenvolvimento sustentável como: saneamento, abastecimento de água, taxa de mortalidade, expectativa de vida, alfabetização de adultos e dióxido de carbono - $\mathrm{CO}_{2}$. A intenção é verificar diferentes dimensões ambientais e sua forma de relação com o crescimento, a partir da heterogeneidade de países observados, ao mesmo tempo em que se testa a hipótese de convergência a nível mundial.

$\mathrm{O}$ artigo se encontra divido em seis seções, incluindo esta introdução. A segunda fundamenta a relação entre "poluição e crescimento"; a terceira aborda brevemente os elementos envolvidos no desenvolvimento sustentável e as suas dimensões a serem testadas; a quarta apresenta o modelo utilizado e a base de dados; a quinta discute os resultados empíricos obtidos e a última seção é dedicada às conclusões.

\section{Meio Ambiente e Crescimento}

O estudo da relação entre crescimento econômico e meio ambiente não é novo na literatura de crescimento econômico e remonta ao final da década de 1960 e início da de 1970. Por um lado, o impacto do crescimento sobre o meio ambiente foi observado por Mishan (1969), Solow (1972) e Commoner (1972). De outro, a forma como o crescimento econômico se processa, considerando diferentes aspectos das limitações impostas pelo meio ambiente, foi discutido por Forster (1973) e Gruver (1976), os quais enfocaram a presença de efeitos ambientais indesejáveis e a conseqüente introdução do controle da poluição no modelo de crescimento neoclássico; o efeito da geração de resíduos 
foi tratado por D’Arge (1971), D’Arge e Kogiku (1973); e quanto à utilização dos recursos naturais, Heal e Dasgupta (1975), Stiglitz (1975), Smith (1976), Anderson (1972) e Kamien Schwartz (1978) foram os primeiros a abordar a questão.

A primeira evidência empírica da existência de uma curva ambiental de Kuznets foi apresentada por Grossman e Krueger (1993), os quais mostraram evidências para os EUA de que a poluição do ar urbano teria um ponto de inflexão da renda ao qual os poluentes - material particulado em suspensão (SPM) e dióxido sulfúrico $\left(\mathrm{SO}_{2}\right)$ - tornavam-se, a partir daquele ponto, função decrescente da renda.

Daí em diante, outros estudos empíricos podem ser citados como sustentando a hipótese da Curva Ambiental de Kuznets. Selden e Song (1993) mostraram, usando emissões de poluentes do ar (monóxido de nitrogênio - NO e monóxido de carbono - CO), que existe substancial suporte para assegurar a hipótese de "U-invertido", mesmo considerando medidas de poluição mais agregadas, em contraponto às medidas usuais de concentrações atmosféricas urbanas. Similarmente, Hettige et al. (1992) já havia demonstrado resultado similar considerando medidas de emissões tóxicas mais abrangentes. Além desses, outros estudos empíricos como Shafik (1994), Cropper e Griffiths (1994), Hilton e Levinson (1998) podem ser citados para corroborar a hipótese da curva ambiental de Kuznets. Não obstante, as explicações para essas evidências parecem não ser objeto de consenso entre os economistas.

Mais recentemente, Harbaugh et al. (2000), usando a mesma base de Grossman e Krugger (1993), com algumas modificações, chegaram a resultados contraditórios aos destes, de forma que evidenciaram que existe pouco suporte empírico para a sustentação da curva ambiental de Kuznets.

Apesar dessas evidências empíricas, vale aqui citar a observação de Ros (2000), segundo o qual a curva de Kuznets é baseada em um controverso fenômeno empírico sem uma plausível e geralmente aceita explicação teórica. De fato, essa observação pode, em certo grau, ser estendida à relação entre crescimento e poluição, embora não exista consenso quanto às experiências dos países em desenvolvimento replicarem a relação côncava da curva de Kuznets. Muito embora as explicações teóricas consigam uma boa aceitação quanto à fase ascendente 
dessa curva, não há convencimento suficiente para sua porção descendente, em que pesem os fatores causais que a determinam.

Sobre essa questão, uma das formulações teóricas é proposta por Andreoni e Levinson (1998), onde mostraram que a curva ambiental de Kuznets pode ser derivada simplesmente da característica tecnológica do esforço gasto para se diminuir a poluição. Assim, se a tecnologia de produção para diminuir a poluição exibir retornos crescentes de escala, essa seria uma condição suficiente para que se pudesse apresentar uma relação entre crescimento e poluição na forma de um "U” invertido.

Em termos mais gerais, pode-se pensar que à medida que as economias evoluem de uma fase essencialmente agrícola para uma fase de industrialização e modernização da agricultura haveria uma correlação positiva com o aumento da emissão de poluentes. Entretanto, outros fatores são apontados como amortecedores ou compensadores desse processo, tal que a partir de um certo ponto observa-se uma inflexão para baixo dessa relação. Segundo Selden e Song $(1994,1995)$ esses fatores seriam: i) elasticidade-renda positiva para qualidade ambiental; ii) mudanças na composição da produção e consumo; iii) aumento do nível educacional e consciência ambiental; e iv) sistemas políticos mais abertos. Segundo esses autores, a trajetória da relação entre desenvolvimento e poluição parece refletir tanto forças de mercado quanto de uma regulamentação ambiental mais exigente, como aquelas observadas nos países desenvolvidos. Ou seja, o próprio desenvolvimento tecnológico implica tanto em aumento de produtividade, como também adoção mais intensa de tecnologias cada vez mais "limpas", estas em decorrência da imposição de leis ambientais e exigências do mercado externo.

A relação positiva entre crescimento da renda e poluição é bem intuitiva. Indivíduos com maior renda consomem mais e, portanto, criam, inevitavelmente, mais poluição através da geração de resíduos sólidos per capita, bem como geram mais poluentes atmosféricos, devido à maior utilização de eletro-domésticos, veículos, entre outros. Por seu turno, com o crescimento da demanda, as firmas produzem mais e, conseqüentemente, na média, produzem mais poluentes atmosféricos e da água, assim como uma maior quantidade de resíduos industriais. Por outro lado, entender a fase declinante da curva de Kuznets é, todavia, menos intuitiva, a não ser que se aceite a priori que a cada nova 
tecnologia embuta a característica de ser menos poluente. Mas, isto não acontece de forma homogênea em todos os setores de uma economia, a menos que, como sugerem Suri e Chapman (1998), as economias avançadas exportassem seus processos de produção intensivos em poluição para os países menos desenvolvidos.

Andreoni e Levinson (1998) contestam essa segunda explicação ao observarem que esse processo de melhora ambiental não seria indefinidamente replicável, uma vez que os países mais pobres ao se desenvolverem não poderiam utilizar desse artifício.

Uma explicação alternativa para que os países desenvolvidos possam exibir a fase decrescente da curva ambiental de Kuznets foi buscada no campo institucional por Jones e Mannueli (1995). Eles argumentam que as instituições tomadoras de decisões na área ambiental, nos países desenvolvidos, seriam as responsáveis pela internalização das externalidades ambientais e, portanto, responsáveis pela diminuição da poluição gerada nesses países. A qualidade dessas instituições poderia, inclusive, gerar um formato contrário que fosse diferenciado do representado pela curva ambiental de Kuznets.

Uma contribuição que busca a conexão entre a evidência empírica e a teoria da mudança tecnológica e crescimento econômico, num contexto do modelo "quality leader" de Grossman e Helpman (1991), é apresentada por Smulder e Bretschger (2001), onde a curva de Kuznets é explicada em um modelo de crescimento endógeno a partir de três elementos chaves. Primeiro, a mudança tecnológica como capaz de permitir reduções na poluição; segundo, a mudança intra-setorial para acompanhar a introdução de tecnologias redutoras de poluição; terceiro, mudanças técnicas intra-setoriais como decorrentes de mudanças políticas e tomada de consciência ambiental. Nesse contexto, é possível se distinguir quatro fases na formação da curva ambiental de Kuznets. A primeira é chamada pelos autores de "fase verde", na qual a produção usa uma tecnologia geral que não causa poluição. Na segunda fase, é introduzida gradualmente na economia uma tecnologia geral que permite a produção a um custo de trabalho mais baixo. Esta nova tecnologia é poluidora, mas a poluição causada pelas firmas não é ainda de conhecimento público. Esta é a denominada "fase de confiança”. A terceira fase se inicia após ter sido revelada publicamente que a nova tecnolo- 
gia é prejudicial em termos ambientais. Assim, com o crescimento da poluição, dá-se início a "fase de alarme". Nessa fase o Governo cria impostos ambientais e taxa as firmas geradoras de poluição, o que leva à conformação de uma nova tecnologia geral que seja não poluidora. Com a implantação dessa nova tecnologia, surge a denominada "fase limpeza”. Dessa forma, a tecnologia limpa é gradualmente introduzida em diferentes setores da economia e a poluição decresce no curso do tempo. A partir da segunda fase, existem subfases em que a pesquisa é primeiramente utilizada para gerar uma nova tecnologia e depois se desloca para a melhoria da variedade dos produtos existentes.

Uma vez expostas as argumentações teóricas e evidências da literatura relacionando crescimento e ciclo ambiental, faz-se em seguida uma conexão desta relação com o desenvolvimento sustentável. Tal conexão justifica-se porque, em primeiro lugar, crescimento pode não implicar necessariamente em desenvolvimento econômico. Segundo, insere-se o meio ambiente para dar sustentação ao desenvolvimento, o qual tem por base os indicadores sociais, e atende ao que se propõe no presente artigo.

\section{Desenvolvimento Sustentável}

A idéia do desenvolvimento sustentável foi originalmente pensada pela União Internacional para a Conservação da Natureza (UICN), em 1980, e depois consagrada internacionalmente pelo Relatório "Nosso Futuro Comum" da Comissão Mundial sobre o Meio Ambiente e Desenvolvimento, em 1986, e Agenda 21 (1992), esse último documento já em caráter mais normativo e multilateral.

Na última década sua difusão e conteúdo, entretanto, deveu-se em grande parte aos esforços do Programa das Nações Unidas para o Meio Ambiente (PNUMA), Banco Mundial, através do seu Departamento de Meio Ambiente, além de entidades não-governamentais como World Wild Fund (WWF) e WorldWatch Institute. Assim, a despeito da diversidade e abrangência que esse conceito tomou, inclusive suscitando diferentes conotações e interpretações, referências ao desenvolvimento sustentável devem levar em conta pelo menos dois eixos objetivos fundamentais: 
1. Atingir um nível de bem-estar econômico-social adequado e eqüitativamente distribuído;

2. Utilizar os recursos naturais de maneira a se garantir a integridade ecológica, o que significa seu uso racional intertemporal.

Esses dois eixos podem ser melhor especificados, tal que resultam em três objetivos críticos:

i) Melhoria da qualidade de vida da população - significando a garantia da satisfação de suas necessidades essenciais como alimento, energia, água, saneamento básico;

ii) Alcance da equidade social - implicando na garantia de iguais oportunidades aos indivíduos de uma sociedade a serviços como educação, saúde, justiça entre outros;

iii) Harmonia na exploração do meio natural entre as gerações presente e futura - garantindo a manutenção de um padrão tecnológico que respeite os limites da sustentabilidade ecológica, tanto com relação ao uso racional dos recursos naturais pelo processo produtivo, como também quanto aos efeitos ambientais gerados por esse processo;

Sob esses objetivos críticos, é que um conjunto de estudos comparativos entre países tem sido realizado, em especial, o Human Development Report, publicado anualmente pelo PNUD, tal que se pode inferir, através de alguns indicadores - entre eles, o Índice de Desenvolvimento Humano (IDH) -, como os países têm evoluído para atingir o desenvolvimento sustentável. Sendo assim, procurou-se verificar como ocorre a relação entre o crescimento, medido pela evolução do Produto Interno Bruto (PIB) e seu impacto sobre o desenvolvimento sustentável, tomando as seguintes dimensões:

Saúde $\rightarrow\left\{\begin{array}{l}\text { Expectativa de vida } \\ \text { Taxa de mortalidade }\end{array} \quad\right.$ Infra - Estrutura $\rightarrow\left\{\begin{array}{l}\text { Déficit em saneamento } \\ \text { Déficit de água potável }\end{array}\right.$ Poluição $\rightarrow\left\{\begin{array}{l}\text { Emissões de } \mathrm{CO}_{2} \\ \text { Material particulado }\end{array} \quad\right.$ Educação $\rightarrow$ \{́éficit na alfabetização de adultos

\section{Metodologia}

A equação básica utilizada para verificação empírica da hipótese original de Kuznets, depois modificada para se testar a curva ambiental de Kuznets, é especificada na forma cúbica a seguir: 


$$
G I N I_{i t}=\beta_{0}+\beta_{1} P I B C_{i t}+\beta_{2} P I B C_{i t}^{2}+\beta_{3} P I B C_{i t}^{3}+D R_{i t}+\varepsilon_{i t}
$$

onde, $G I N I$ = coeficiente de Gini; PIBC = PIB per capita expresso na paridade do poder de compra; $D R=$ dummy regional, assumindo o valor " 0 " para os países da África-Subsahara e valor "1" para os demais; $i$ e $t$ referem-se ao país e o tempo, respectivamente.

A modificação básica para a inspeção da curva ambiental de Kuznets é feita na variável dependente, que é substituída por um indicador ambiental, geralmente uma medida de poluição hídrica ou atmosférica. No caso específico deste estudo, assumem-se várias variáveis dependentes, cada uma relacionada com o desenvolvimento sustentável, seguindo as dimensões apresentadas na seção anterior. A variável independente chave, por seu turno, continua sendo o PIB per capita, acrescida da variável dummy, onde nesta definição está implícito o pressuposto de que a região da África Subsahariana, por ser a região que apresenta o maior número de países com indicadores baixos, inclusive o Índice de Desenvolvimento Humano (IDH), portanto, não teriam esses países atingido os estágios mais avançados de proteção ambiental, como discutido em seção anterior.

O modelo especificado na forma linear dos parâmetros, e caso possuísse erro esférico, permitiria, em primeira instância, o uso do método dos Mínimos Quadrados Ordinários (MQO), tendo como sinais esperados positivo para $\beta_{1}$, negativo para $\beta_{2}$ e, caso se confirme a rejeição da hipótese da curva ambiental de Kuznets, positivo para $\beta_{3}$.

A especificação adotada na equação (1) está sujeita ao problema da variável omitida do modelo, que pode trazer severos danos estatísticos às estimativas. Dessa forma, com o intuito de dirimir o possível problema da endogeneidade daí decorrente, uma solução que daria robustez ao modelo seria a utilização de dados em painel.

Na suposição de que as variáveis omitidas variam entre os países, mas não no tempo, o modelo mais adequado a ser adotado é o do efeito fixo. Isto significaria que a variável omitida poderia ser tratada como um parâmetro a ser estimado em cada observação cross- section.

O modelo do efeito fixo parte do princípio que a variável omitida é não correlacionada com cada uma das variáveis explicativas em todos os perío- 
dos. Se isso não ocorre, então qualquer transformação que elimine a variável omitida gerará estimativas deficientes. Todavia, se não existe correlação entre a variável omitida e qualquer das variáveis explicativas do modelo, então a estimação mais adequada é o modelo de efeito aleatório.

\subsection{Dados}

Com o intuito de introduzir as dimensões ambientais especificadas na seção 3, que vão ao encontro da noção de desenvolvimento sustentável, foram testadas as seguintes variáveis: i) proporção da população sem abastecimento de água, isto é, o déficit em seu fornecimento; ii) proporção da população sem acesso à rede sanitária, ou seja, o déficit em seu fornecimento; iii) déficit na expectativa de vida em relação ao ideal; iv) déficit na alfabetização de adultos com relação ao ideal; v) emissão de dióxido de carbono per capita $\left(\mathrm{CO}_{2}\right)$; vi) taxa de mortalidade.

As duas primeiras variáveis foram expressas como um déficit, isto é, considerando diretamente a proporção da população do país que não tem acesso àquele indicador desejável de desenvolvimento sustentável. As duas seguintes são definidas considerando a sua condição relativa a um nível ideal. As demais variáveis, emissão de dióxido de carbono $\left(\mathrm{CO}_{2}\right)$ e taxa de mortalidade, foram consideradas em termos absolutos, uma vez que ambas são expressas como uma taxa que reduz o bem-estar. A variável $\mathrm{CO}_{2}$ descreve o total de toneladas métricas per capita emitida pela população residente do país e a taxa de mortalidade refere-se ao número de óbitos de crianças até completar um ano dentre mil nascidos vivos.

A variável expectativa de vida foi calculada a partir do país com a maior expectativa de vida para aquele ano (2000), modelo cross section, no caso o Japão, cuja longevidade média da população era de 81 anos. Assim, o déficit dos outros países foi calculado como a diferença à média atingida pelo Japão em termos proporcionais. Com relação à extensão para dados em painel, tomando-se os anos de 1980, 1985, 1990, 1995 e 2000, o Japão continuou sendo o país de maior longevidade e, portanto, aquele que serviu como base de referência. De maneira semelhante, o déficit de alfabetização de adultos denota a diferença do percentual de adultos alfabetizados do país em relação a $100 \%$.

Os dados utilizados foram provenientes basicamente do Human De- 
velopment Report do PNUD e World Development Indicators do Banco Mundial. Estes, por sua vez, utilizam como fonte original de informações, além da base de dados gerada por diversos órgãos da ONU, como UNESCO, por exemplo, aqueles disponibilizados pelo próprio Banco Mundial, e outros bancos de dados internacionais como Penn World Table, de Alan Heston e Robert Summers. O ano de referência foi o de 2000, com exceção das informações do dióxido de carbono (1998).

\section{Resultados}

\subsection{Modelo Cross - Section}

Os resultados do modelo cross-section são apresentados na Tabela 2 , onde se verificam diferentes interpretações para a comprovação ou não de uma curva de Kuznets . É importante observar que todas as variáveis denotam um déficit ou algo negativo para o desenvolvimento sustentável de modo que o sinal positivo da variável PIBC e negativo para $\mathrm{PIBC}^{2}$, seguido ou não de um sinal positivo para a variável $\mathrm{PIBC}^{3}$, é a evidência necessária para a existência da curva de Kuznets. Isso foi evidenciado apenas para as variáveis taxa de mortalidade e $\mathrm{CO}_{2}$, onde esta última variável, entretanto, não mostrou seu coeficiente ser significante. Todas as demais variáveis apresentaram evidências para a forma de "U” e não "U-invertido".

Observa-se que, a partir do teste Breusch-Pagan/Cook Weisberg (coluna 9 da Tabela 2), verificou-se que somente as equações para a expectativa de vida e déficit de abastecimento de água, apresentavam erros homocedásticos. Assim sendo, no sentido de fazer a correção devida, as demais equações foram reestimadas, considerando erros robustos para heterocedasticidade. Esses são os resultados mostrados na Tabela 2 para as equações: déficit no saneamento, taxa de mortalidade, déficit na taxa de analfabetismo e $\mathrm{CO}_{2}$.

\subsection{Correlação das Variáveis}

Poder-se-ia inquirir sobre se as variáveis com relação ao GDP per capita apresentam um formato próximo à curva de Kuznets, então, qual 
seria a possível relação de causalidade dessas variáveis entre si, que poderia causar danos econométricos. Neste sentido, com o intuito de identificar algum tipo de colinearidade entre as variáveis dependentes utilizadas, procedeu-se o cálculo da matriz de correlação (Tabela 1).

Ressalte-se a correlação positiva entre $\mathrm{CO}_{2}$ per capita e taxa de mortalidade, como era de se esperar. Também como esperado, há correlação positiva entre o déficit da provisão de água e de saneamento com a taxa de mortalidade, bem como dessas duas variáveis com a expectativa de vida, o que sugere que esses déficits evoluem na mesma direção.

Tabela 1 - Correlação das Variáveis de Desenvolvimento Sustentável

\begin{tabular}{l|c|c|c|c|c|c}
\hline & Alfabetização & Mortalidade & Saneamento & Água & $\mathrm{CO}_{2}$ & $\begin{array}{c}\text { Exp. } \\
\text { Vida }\end{array}$ \\
\hline Alfabetização & 1 & & & & & \\
\hline Mortalidade & $-0,0179$ & 1 & & & & \\
\hline Saneamento & 0,0360 & 0,0365 & 1 & & & \\
\hline Água & $-0,0366$ & 0,0365 & 0,0805 & 1 & & \\
\hline $\mathrm{CO}_{2}$ & $-0,0251$ & 0,0693 & $-0,0139$ & $-0,0614$ & 1 & \\
\hline Exp. Vida & 0,8043 & $-0,256$ & 0,0162 & 0,0683 & $-0,0752$ & 1 \\
\hline
\end{tabular}

Embora sabendo-se a priori que os países de baixa renda são aqueles que apresentam os maiores déficits para com o desenvolvimento sustentável seja para qualquer indicador tomado como referencial neste estudo. Pelas baixas correlações apresentadas a Tabela 1 revela uma fraca possível relação de causalidade entre as variáveis.

\subsection{Resultados dos Modelos com Dados em Painel}

Os resultados para dados em painel, para as variáveis selecionadas, confirmam aqueles do modelo cross-section, tanto em relação às regressões do modelo com efeito fixo (Tabela 3) quanto para o modelo com efeito aleatório (Tabela 4). Exceção é feita para com a variável taxa de mortalidade, que apresentou nesses dois últimos modelos um formato de "U” e não "U-invertido", ao contrário daquele observado no modelo cross-section. Isto denota que a introdução da dimensão tempo, conduz a uma modificação do efeito do PIB per capita sobre a taxa de mortali- 


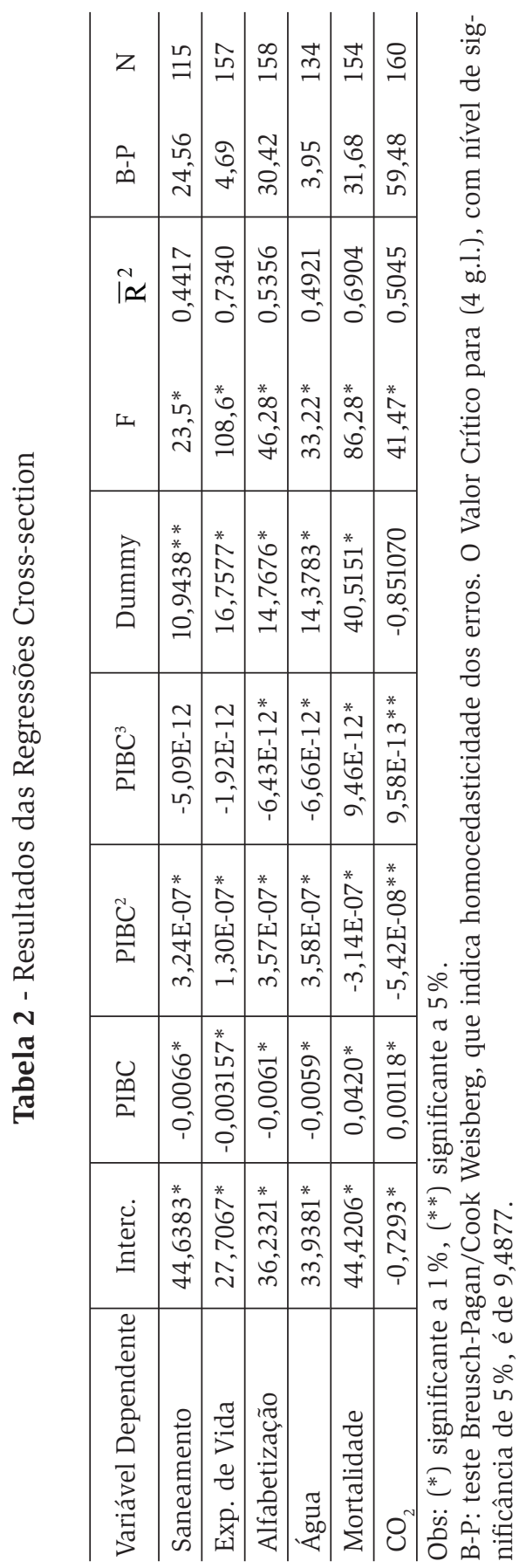

RER, Rio de Janeiro, vol. 44, no 03, p. 525-547, jul/set 2006 - Impressa em setembro 2006 


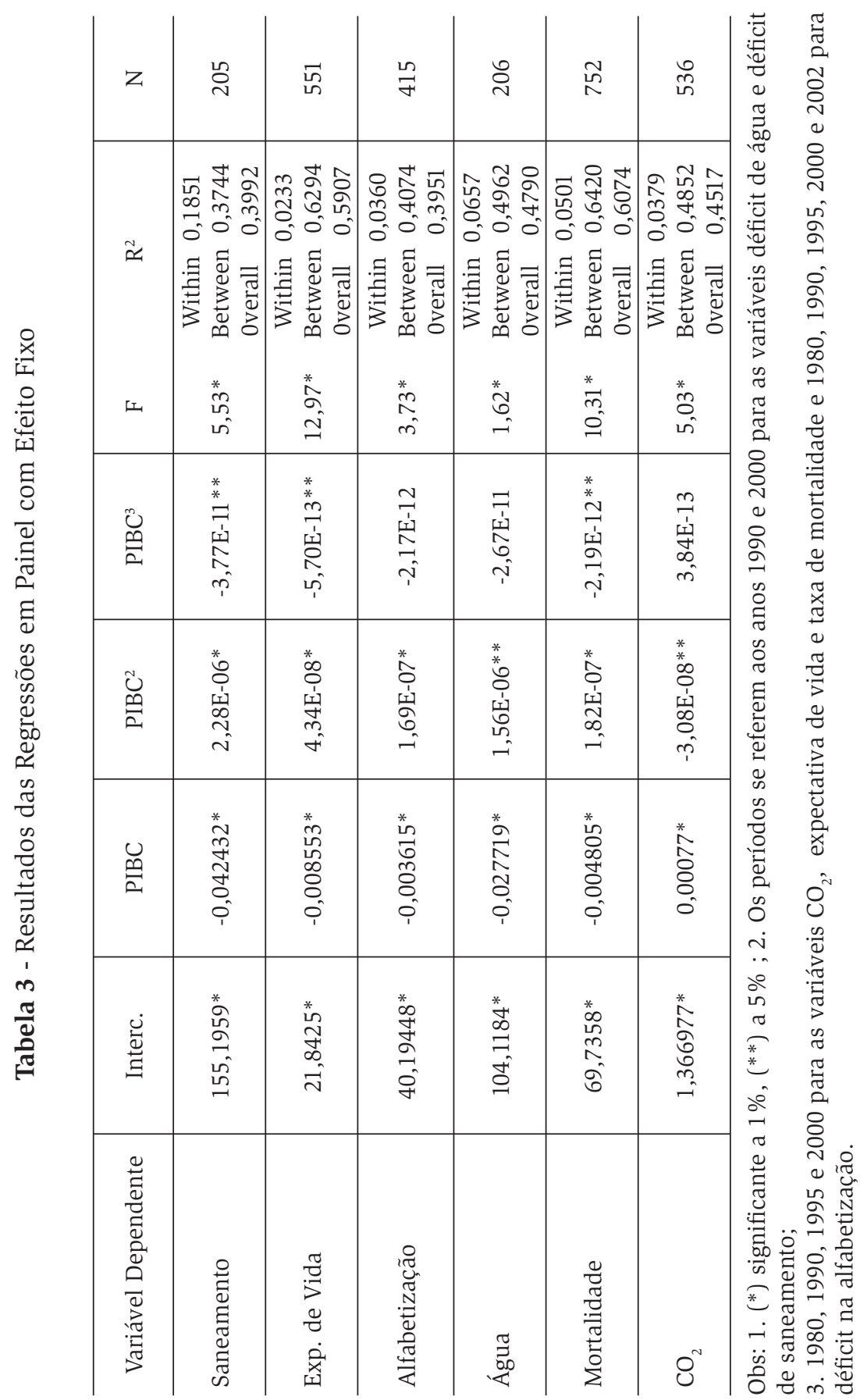

RER, Rio de Janeiro, vol. 44, no 03, p. 525-547, jul/set 2006 - Impressa em setembro 2006 
dade. Ou seja, os países sustentam um crescimento e depois um decrescimento com o PIB per capita que, ao longo do tempo, faz essa tendência se inverter, tal que se evidencia uma queda e depois um aumento da taxa de mortalidade em função do crescimento do PIB per capita.

Além disso, destaca-se também o comportamento do $\mathrm{R}^{2}$ em todas as regressões nos modelos de efeito fixo e efeito aleatório, refletindo na significância das regressões via o teste F.

Tabela 4 - Resultados das Regressões em Painel com Efeito Aleatório

\begin{tabular}{|c|c|c|c|c|c|c|}
\hline \multirow{2}{*}{$\begin{array}{l}\text { Variáveis } \\
\text { Explicativas }\end{array}$} & \multicolumn{6}{|c|}{ Variáveis Dependentes } \\
\hline & Saneam. & $\begin{array}{l}\text { Exp. de } \\
\text { Vida }\end{array}$ & $\begin{array}{l}\text { Alfabet. de } \\
\text { Adultos }\end{array}$ & $\begin{array}{l}\text { Abast. de } \\
\text { Água }\end{array}$ & $\begin{array}{l}\text { Tx. de Mor- } \\
\text { talidade }\end{array}$ & $\mathrm{CO}_{2}$ \\
\hline Intercepto & $52,5800^{*}$ & $23,4063^{*}$ & $39,1062 *$ & $38,4751^{*}$ & $72,2358^{*}$ & $-0,6539$ \\
\hline PIBC & $-0,0086^{*}$ & $-0,0023^{*}$ & $-0,0054^{*}$ & $-0,0064^{*}$ & $-0,0086^{*}$ & $0,0012^{*}$ \\
\hline $\mathrm{PIBC}^{2}$ & $4,6 \mathrm{E}-07^{*}$ & 9,9E-08* & $2,8 \mathrm{E}-07^{*}$ & $3,5 \mathrm{E}-07^{*}$ & $3,7 \mathrm{E}-07^{*}$ & $-4,7 \mathrm{E}-08^{*}$ \\
\hline $\mathrm{PIBC}^{3}$ & $-8,1 \mathrm{E}-12 *$ & $-1,2 \mathrm{E}-12^{*}$ & $-3,9 \mathrm{E}-12 *$ & $-6,13 \mathrm{E}-12^{*}$ & $-4,9 \mathrm{E}-12^{*}$ & $6,1 \mathrm{E}-13^{*}$ \\
\hline Dummy & 6,7626 & $18,5826^{*}$ & $19,9148^{*}$ & $11,7214^{*}$ & $44,5332 *$ & $-0,0075$ \\
\hline \multicolumn{7}{|l|}{ Estatísticas } \\
\hline$\chi^{2}$ & $58,63^{*}$ & $619,44^{*}$ & $119,20^{*}$ & $120,20^{*}$ & $534,06^{*}$ & $141,75^{*}$ \\
\hline \multirow{3}{*}{$\mathrm{R}^{2}\left\{\begin{array}{l}\text { Within } \\
\text { Between } \\
\text { Overall }\end{array}\right.$} & 0,178 & 0,0148 & 0,0345 & 0,0642 & 0,0489 & 0,0357 \\
\hline & 0,385 & 0,8162 & 0,4994 & 0,5463 & 0,7671 & 0,5030 \\
\hline & 0,403 & 0,7979 & 0,4700 & 0,5259 & 0,7318 & 0,4662 \\
\hline $\mathrm{N}$ & 205 & 551 & 415 & 206 & 752 & 536 \\
\hline
\end{tabular}

Obs: (*) Significante a $1 \%$. Os períodos se referem aos anos de 1990 e 2000 para as variáveis déficit de água e déficit de saneamento; 1980, 1990, 1995 e 2000 para as variáveis $\mathrm{CO}_{2}$, déficit de expectativa de vida e taxa de mortalidade e 1980, 1990, 1995, 2000 e 2002 para a variável déficit na alfabetização.

A inspeção acerca do melhor modelo, se com efeito fixo ou com efeito aleatório, é realizada mediante a aplicação do Teste de Hausman. Assim, considerando os resultados obtidos do teste para cada variável dependente, apresentados na Tabela 5, pode-se inferir que melhor modelo de ajuste é aquele com efeito fixo para todas as variáveis, com exceção da variável déficit no abastecimento de água. 
Tabela 5 - Teste de Hausman

\begin{tabular}{l|c}
\hline \multicolumn{1}{c|}{ Variável Dependente } & Teste de Hausman*: $\boldsymbol{\chi}^{2}$ \\
\hline Saneamento & 9,18 \\
\hline Exp. de Vida & 44,16 \\
\hline Alf. de Adultos & 4,79 \\
\hline Abast. de Água & 2,76 \\
\hline Taxa de Mortalidade & 30,13 \\
\hline $\mathrm{CO}_{2}$ & 9,02 \\
\hline
\end{tabular}

* Valor crítico a $5 \%$ com $(\mathrm{K}=1)$ é igual a 3,84 .

\subsection{Convergência}

Com o intuito de preencher uma lacuna na literatura, tal como sugerida por Brock e Taylor (2004), buscou-se analisar a existência ou não de convergência dos indicadores de desenvolvimento entre os países, com base nas condições necessária e suficiente para tal ocorrência.

Inicialmente, verifica-se, a partir da equação que exprime a condição necessária para convergência tradicional, o comportamento de $\beta$ -Convergência, usada tanto para designar a ocorrência de convergência condicional quanto para a convergência incondicional. Neste caso, a idéia aqui é verificar se esta condição necessária de convergência é confirmada para os indicadores entre os países mais e menos desenvolvidos, ou seja, se alguns indicadores mudam ao longo do tempo a taxas diferenciadas entre os países. A equação de $\beta$-Convergência é especificada como:

$$
\ln \left(\mathrm{y}_{\mathrm{i}, \mathrm{j}, \mathrm{t}} / \mathrm{y}_{\mathrm{i}, \mathrm{j}, \mathrm{t}+\mathrm{T}}\right)=\alpha_{\mathrm{i}}+\beta_{i} \ln \left(\mathrm{y}_{\mathrm{i}, \mathrm{j}, \mathrm{t}+\mathrm{T}}\right)+\varepsilon_{\mathrm{ij}}
$$

onde, $\mathrm{y}_{\mathrm{i}, \mathrm{j}, \mathrm{t}}$ denota o indicador de desenvolvimento "i" do país " $\mathrm{j}$ " nos períodos $\mathrm{t}=1980$ e $\mathrm{t}+\mathrm{T}=2000 ; \varepsilon_{i j}$ é o erro estocástico. As estimativas corrigidas por heterocedasticidade se detectada no teste de White, estão dispostas na Tabela 6. 
Tabela 6 - Estimativas da Equação (2)

\begin{tabular}{|c|c|c|c|c|c|c|}
\hline \multirow{2}{*}{$\begin{array}{l}\text { Coef. } \\
\text { Estimado }\end{array}$} & \multicolumn{7}{|c|}{ Variáveis } & Dependentes \\
\cline { 2 - 7 } & Saneam. & Exp.vida & Analf. & Água & Mort. & $\mathrm{CO}_{2}$ \\
\hline$\hat{\alpha}_{i}$ & -0.8411 & 0.769584 & 1.1425 & $-0.3025^{*}$ & 1.3616 & $-0.0436^{*}$ \\
\hline$\hat{\beta}_{i}$ & 0.1732 & -0.198476 & -0.1912 & $0.0510^{*}$ & -0.2421 & -0.1226 \\
\hline $\mathrm{R}^{2}$ & 0,35 & 0,27 & 0,55 & $0,02^{*}$ & 0,49 & 0,06 \\
\hline
\end{tabular}

(*) Não significante; os demais coeficientes são significantes a, no máximo, $1 \%$.

A verificação da condição suficiente para convergência dos indicadores foi baseada em uma estatística simples, porém eficaz, o coeficiente de variação:

$$
\mathrm{CV}_{\mathrm{i}}=\hat{\sigma}_{\mathrm{i}} / \overline{\mathrm{y}}_{\mathrm{i}}
$$

onde, $\mathrm{CV}_{\mathrm{i}}$ é o coeficiente de variação do indicador "i"; $\hat{\sigma}$ é o desvio padrão amostral e $\bar{y}_{i}$ a média simples. Observa-se que sua redução ao longo do tempo aponta para o atendimento da condição, desde que a condição necessária seja atendida. Os resultados encontram-se na Tabela 7.

Tabela 7 - Coeficientes de Variação dos Indicadores de Desenvolvimento, $1980 / 2000$

\begin{tabular}{|c|c|c|c|c|c|c|}
\hline \multirow{2}{*}{ Anos } & \multicolumn{7}{|c|}{ Indicadores } \\
\cline { 2 - 7 } & Saneam. & Exp.vida & Analf. & Água & Mort. & $\mathrm{CO}_{2}$ \\
\hline 1980 & 0,4073 & 0,1794 & 0,9904 & 0,1186 & 0,7138 & 1,6778 \\
\hline 2000 & 0,3477 & 0,1910 & 0,8774 & 0,1141 & 1,0388 & 1,6630 \\
\hline $\mathrm{N}$ & 75 & 159 & 100 & 74 & 155 & 155 \\
\hline
\end{tabular}

Desde que o sinal negativo da estimativa de $\beta_{i}$ indica o atendimento da condição necessária de convergência, os resultados da Tabela 6 revelam a não convergência para os indicadores saneamento, água potável e emissões de $\mathrm{CO}_{2}$. Dos indicadores restantes, apenas déficit de analfabetos atende as condições necessária e suficiente de convergência. Isso indica o acerto de os países pobres imprimirem severos esforços na melhoria educacional, pois do ponto de vista teórico e empírico, capital 
humano, tendo como proxy educação, é a variável endógena mais importante para gerar crescimento econômico. (Barro, 2000; Grossman, 2001).

\section{Conclusão}

Pode-se extrair algumas conclusões importantes deste estudo que contribui para o entendimento do desenvolvimento sustentável a nível mundial. A primeira é que as variáveis que denotam desenvolvimento sustentável, dentro da definição aqui tomada, apresentam uma relação com renda per capita dos países que pouco se aproximam do que é descrito na literatura como Curva Ambiental de Kuznets, isto é, tomando a forma de U-invertido. Este comportamento seria descrito apenas para as variáveis $\mathrm{CO}_{2}$ e taxa de mortalidade no modelo "cross section" e $\mathrm{CO}_{2}$ no modelo com dados em painel. As demais variáveis selecionadas apresentam um formato exatamente contrário, isto é, um formato de "U" ou senoidal, e não de "U-invertido", revelando um comportamento que mostra que os déficits decrescem com a renda até um determinado nível, para depois aumentar, ou senão movimentar-se ciclicamente. Tal resultado parece refletir o fato de as variáveis terem efeitos locais mais destacados, bem como dependerem de forma mais direta de políticas sociais internas, ou serem aquelas que não têm evoluído na direção desejável. Ao contrário do que ocorre com a variável $\mathrm{CO}_{2}$ que apresenta um comportamento sujeito a um maior monitoramento internacional, bem como dos acordos normativos na busca do desenvolvimento sustentável.

Em princípio esse resultado revelaria um comportamento entre os países, onde suas economias não estariam em consonância com o desenvolvimento sustentável, pelo menos em seu conjunto. Os coeficientes de correlação dos indicadores apontam que os países que apresentam déficit para com o desenvolvimento sustentável o fazem para um conjunto de variáveis que denotam carência para esse estágio de desenvolvimento.

Com base no rigor da observância das condições necessária e suficiente de convergência, contatou-se que o comportamento da dispersão dos indicadores de desenvolvimento sustentável, incluindo a renda, reforça o resultado anterior de que não estaria havendo convergência das economias dos países em direção ao desenvolvimento sustentável, 
exceção feita ao esforço empreendido pelos países mais pobres em aumentar o ritmo de crescimento do nível educacional, em consonância com os preceitos teóricos do crescimento econômico.

\section{Referências bibliográficas}

ANDERSON, Kent P. Optimal Growth When the Stock of Resources is Finite and Depletable. Journal of Economic Theory, v. 4, p.256-267, 1972.

ANDREONI, James; LEVINSON, Arik The Simple Analytics of the Environmental Kuznets Curve, NBER Working Paper, n.6739, 1998.

ARROW, K. et al. Economic Growth, Carrying Capacity and the Environment. Science, v.268, p.520-521, 1995.

BARRO, Robert J. Inequality and Growth in a Panel of Countries. Journal of Economic Growth, v.5, p.5-32, 2000.

BECKERMAN, W. Economic Growth and the Environment: Whose Growth? Whose Environment. World Development, v.20, p.481-496, 1992.

BROCK, W.A; TAYLOR, M.S. The Green Solow Model. NBER Working Paper, n.10557, 2004.

COMMONER, Barry. The Environmental Costs of Economic Growth. In: Robert Dorfman e Nancy Dorfman (orgs). Economics of the Environment Selected Readings, 2 ed, New York, W. W. Norton e Company Inc, 1972 .

CROPPER, M.; GRIFFITHS G. The Interaction of Population, Growth and Environmental Quality. American Economic Review, v.84, p.250254, 1994.

D’ARGE, Ralph C. Essay on Economic Growth and Environmental Quality. Swedish Journal of Economics, v.15, 1971.

D'ARGE, Ralph C.; KOGIKU, K. Economic Growth and the Environment, Review of Economic Studies, v.40, p. 61-77, 1973.

GOODSTEIN, Ebans. Economics and Environment. 2 ed. New York, John Wiley \& Sons, Inc, 1999. 
GROSSMAN, G; KRUEGER. A. Economic Growth and the Environment. Quarterly Journal of Economics, v.110, n.2, p.353-377, 1995.

GROSSMAN, Volker. Inequality, Economic Growth, and Technological Change - New Aspects in an Old Debate. Heidelberg: New York: Physical-Verl, 2001.

GRUVER, Gene W. Optimal Investment in Pollution Control Capital in a Neoclassical Growth Context. Journal of Environmental Economics and Manegement v.3, p.165-177, 1976.

HARBAUGH, William; LEVINSON, Arik; WILSON, David. Reexaming The Empirical Evidence for an Environmental Kuznets Curve. NBER Working Paper 7711, 2000.

HEAL, G.; DASGUPTA, P. The Optimal Depletion of Exhaustible Resources, Review of Economic Studies, Symposium Volume, 1975, p. 3-28.

HILTON, F. G. HANK; LEVINSON, A. Factoring the Environmental kuznets Curve: evidence from automotive lead emissions, Journal of Environmental Economics and Management, v.35, p.126-141, 1998.

JONES, Larry E.; MANUELLI, Rodolfo E. A Positive Model of Growth and Pollution Controls. NBER Working Papers n.5205, 1995.

KHAN, M.E. A Household Level Environmental Kuznets Curve. Economics Letters, v.59, n.2, p.269-273, 1998.

KARMIEN, Morton I; SCHWARTZ, Nancy L. Optimal Exhaustible Resource Depletion with Endogenous Technical Change. Review of Economic Studies, v. 45, p.179-196, 1978.

KUZNETS, Simon. Economic Growth and Income Inequality. American Economic Review, v.45, p.1-28. 1995.

MISHAN, E. J. The Costs of Economic Growth. Ringwood; Vic, Pinguin Australia, 1969.

RAY, Debraj. Development Economics . Princeton, New Jersey, Princeton University Press. 1998.

RUTTAN, Vernon. Tecnology, Growth, and Development: an induced innovation perspective. Oxford University Press, New York, 2001. 
SALA-I-MARTIN, Xavier. The Classical Approach to Convergence Analysis. The Economic Journal, v. 106, p.1019-36, 1996.

SELDEN, Thomas M.; SONG, Daqing. Environmental Quality and Development: is there a Kuznets Curve for air Pollution Emission ?. Journal of Environmental Economics and Management, v. 27, p.147-162, 1994.

SELDEN, Thomas M.; SONG, Daqing. Neoclassical Growth, The J Curve for Abatement and the Inverted U Curve for Pollution. Journal of Environmental and Management, v.29, p.162-168, 1995.

SMITH, Vernon. Control Theory Applied to Natural and Environmental Resources. Journal of Environmental Economics and Management, v. 4, p.1-14, 1997.

SMULDERS, Sjak; BRETSCHGER, Lucas. Explaining Environmental Kuznets Curves: How Pollution Induces Policy and New Technologies. Working paper, Tilburg University, p.25, 2001.

SOLOW, Robert. The Economics of Resources or the Resources of Economics. The American Economic Review, v. 64, n. 2, p.1-14, 1974.

SOLOW, Robert. Intergenerational Equity and Exhaustible Resources. Review of Economic Studies, Symposium Volume, p. 29-45, 1975.

STIGLITZ, Joseph. Growth with Exhaustible Natural Resources: Efficient and optimal Growth Paths, Review of Economic Studies, Symposium Volume, p. 123-52, 1975.

UNDP (United Nations Development Program). Human Development Report 2002: New York: Oxford University Press, 2002.

Recebido em agosto de 2005 e revisto em junho de 2006 